\title{
Aconselhamento Genético
}

\author{
Genetic Counseling
}

\section{Décio Brunoni ${ }^{1}$}

\begin{abstract}
Genetic counseling (GC) is a medical multiprofessional and interdisciplinar health care process. Most of the Brazilian people don't have access to these services. In Brazil there are many GC groups, centers and services in condition to train health care professionals to the Federal Brazilian Public System of Health (Sistema Único de Saúde - SUS). Ministry of Health could easily created an Office of Genetic Services to organize and launch trainning programs. It is also necessary improve genetic educational programs for the health professions as well as educate the public in genetic disorders and birth defects. Support of parent/patient organizations is also desirable. The implementation of such community genetics programs has been recommended by the World Health Organization (WHO).

Key words Genetics, Genetic Counseling, Health Care Delivery Services
\end{abstract}

\section{Resumo}

O Aconselhamento Genético ( $A G$ ) deve ser desenvolvido nas unidades de saúde como um atendimento médico multiprofissional e interdisciplinar. A maioria da população brasileira não tem acesso a esses serviços. Os grupos, centros e serviços de AG existentes no Brasil podem, em curto espaço de tempo, preparar profissionais para atender a rede do Sistema Único de Saúde (SUS). O Ministério da Saúde tem os instrumentos necessários para viabilizar esta estratégia. Medidas que visem incrementar o ensino da genética médica nos cursos de graduação e em curso de especialização/aperfeiçoamento podem ser efetivadas. $\mathrm{O}$ envolvimento da população, de pacientes e famílias no conhecimento dos fatores genéticos que agravam a saúde é desejável e pode ser alcançado por ações de genética comunitária como tem sido recomendado pela Organização Mundial da Saúde (OMS).

Palavras-chave Aconselhamento Genético, Serviços de Genética, Genética e Saúde Pública

\section{I ntrodução}

Uma das definições correntemente aceitas de Aconselhamento Genético (AG) é a adotada pela American Society of Human Genetics (Epstein, 1975). Segundo esta definição, trata-se do processo de comunicação que lida com problemas humanos associados com a ocorrência, ou risco de ocorrência, de uma doença genética em uma família, envolvendo a participação de uma ou mais pessoas treinadas para ajudar o indivíduo ou sua família a: 1) compreender os fatos médicos, incluindo o diagnóstico, provável curso da doença e as condutas disponíveis; 2) apreciar o modo como a hereditariedade contribui para a doença e o risco de recorrência para parentes específicos; 3 ) entender as alternativas para lidar com o risco de recorrência; 4) escolher o curso de ação que pareça apropriado em virtude do seu risco, objetivos familiares, padrões éticos e religiosos, atuando de acordo com essa decisão; 5) ajustar-se, da melhor maneira possível, à situação imposta pela ocorrência do distúrbio na família, bem como à perspectiva de recorrência do mesmo.

Tal definição tem propiciado interpretações diversas do processo e das competências das "pessoas treinadas para ajudar o indivíduo e a família" e quase sempre dá a entender que "os fatos médicos, incluindo o diagnóstico, provável curso da doença e condutas disponíveis"

\footnotetext{
${ }^{1}$ Departamento de Morfologia, Universidade Federal de São Paulo/Escola Paulista de Medicina. Rua Botucatu 740, edifício Leitão da Cunha 04023-900 São Paulo SP. dbrunoni.morf@epm.br
} 
estão plenamente conhecidos. É como se o AG fosse o procedimento pelo qual o paciente e/ou a família viesse finalmente a entender por que foram (ou estão sendo) submetidos a consultas, exames laboratoriais às vezes muito complexos, intervenções de naturezas variadas, cirúrgicas e clínicas, temporárias ou permanentes, com seguimentos e/ou internações hospitalares muitas vezes exaustivos e desgastantes. $E$ que, além de tudo, 0 fato de ter ocorrido com aquele indivíduo ou família abre a possibilidade de vir a recorrer. Neste modelo o aconselhador retém a competência da comunicação para que o paciente e a família entendam o que está acontecendo. É claramente um processo psicoeducacional dinâmico que necessita ser melhor compreendido e pesquisado (Biesecker \& Peters, 2001).

A realidade cotidiana está longe deste modelo. No Brasil, a maioria dos pacientes e famílias acometidos de doenças puramente genéticas ou influenciadas pelos genes desconhecem amplamente a condição médica que possuem e não foram investigados de maneira adequada para evidenciar os fatores genéticos envolvidos. Estamos, portanto, no passo inicial do AG, qual seja o de incrementar medidas que possibilitem um sistema de atendimento pelo qual a maioria da população tenha acesso a serviços e procedimentos que possam revelar a doença genética que possuem. A partir disso, poderão entender sua condição de saúde e as alternativas disponíveis para tratamento e prevenção.

Neste artigo será dada a visão da realidade e perspectivas do AG no serviço assistencial público de saúde brasileiro. Muitas das reflexões e sugestões apresentadas amadureceram nas discussões entre diversos sócios da Sociedade Brasileira de Genética Clínica (SBGC), que, a pedido do Conselho Federal de Medicina (CFM), produziram um documento posicionando-se quanto aos métodos e intervenções no AG (Marques-de-Faria et al., 2000).

\section{O processo do Aconselhamento Genético}

A consulta médica constitui ato imprescindível para o Aconselhamento Genético, pois dela resulta o diagnóstico e, a partir deste, a determinação do prognóstico clínico e reprodutivo em pacientes, indivíduos ou famílias com doenças de etiologia genética ou susceptibilidade geneticamente determinada. A responsabilidade do diagnóstico é do médico, que deve estar familiarizado com o método para atingir tal objetivo, qual seja o da propedêutica genéticoclínica.

Em todas as especialidades médicas (Hampton et al., 1975; Gjorup et al., 1989), e em genética clinica não é diferente (Costa et al., 1985), os pilares da propedêudica são os mais eficazes para se alcançar o diagnóstico: a anamnese e o exame físico. A partir dessas intervenções iniciais, o paciente e/ou a família deverão ser esclarecidos e estimulados a participar do processo. Os exames complementares necessários para firmar ou descartar uma hipótese diagnóstica deverão ser explicados, assim como o significado e alcance dos resultados. Todo o processo e o relacionamento com a equipe de saúde devem ser executados segundo o modelo do consentimento livre e esclarecido.

Com o diagnóstico definitivo, ou não, o paciente e a família começam a ser esclarecidos dos recursos terapêuticos ou, mais freqüentemente, das possibilidades de prevenção primária, secundária ou terciária.

Este evolver de consultas, exames complementares, intervenções, esclarecimentos, apoio e respeito nas decisões, praticados em pacientes e famílias visando ao diagnóstico, tratamento e prevenção de doenças genéticas é o Aconselhamento Genético. Através dele deve-se alcançar o diagnóstico acurado, a identificação de indivíduos em risco clínico e reprodutivo; deve-se assegurar que paciente e família entendem como a herança contribui para a doença e para os riscos de recorrência. É preciso alcançar também a linha de ação que atenda às necessidades e ao ajustamento psicológico e social dos pacientes e famílias. Além de tudo isso, em muitas situações, o paciente e a família devem entender que o diagnóstico de uma condição genética implica riscos, benefícios, limitações e conseqüências psicológicas e econômicas (Pergament, 1997). 
Como em qualquer outro ato médico complexo, exige uma abordagem multiprofissional e interdisciplinar. Além da competência específica que a profissão lhes atribui, todos os profissionais envolvidos devem ter recebido formação em genética médica básica e treinamento nos procedimentos e intervenções sob sua responsabilidade. Há necessidade de que estes profissionais contem com equipe de apoio psicossocial. Elas são fundamentais para garantir que os pacientes e famílias tenham melhor entendimento sobre o processo, o que otimizará a aderência necessária.

Uma visão com tal compreensão do AG foi referendada pelo grupo de especialistas em genética humana, médica e clínica que redigiu o documento "Conclusiones y recomendaciones de la reunión de expertos em servicios de genética médica em América Latina", em reunião durante o 9o Congresso Internacional de Genética Humana, realizado no Rio de Janeiro em 1996 (Grupo de Trabalho, 1997).

\section{O AG no Brasil: estado atual}

A partir dos anos 60 e 70, serviços de AG começaram a se desenvolver de maneira mais consistente, com interesse maior na pesquisa de certas doenças ou grupos de doenças genéticas. Esses serviços quase sempre estavam ligados a cursos de Pós-Graduação em Genética Humana e/ou Médica. Durante as décadas de 1970 e 1980, foram se estruturando serviços de AG com maior capacidade assistencial (Brunoni, 1997, 1999). Esses serviços são quase todos ligados a hospitais e/ou instituições universitárias e, em recente cadastramento realizado pela SBGC, foram catalogados 64 serviços. Deles, 85\% localizam-se no sul/sudeste, sendo que mais da metade do total apenas em dois estados (RS e SP) (Marques-de-Faria et al., 2001).

O número de procedimentos e pessoal envolvido com AG no Brasil foi avaliado em 1997, sendo considerado insignificante (Brunoni, 1997). Pior, estima-se que intervenções de AG não são praticadas na grande maioria dos pacientes e famílias que padecem de doenças genéticas.

Isso significa que a maioria desses pacientes é atendida pelo sistema de saúde e não tem sua condição diagnosticada e/ou esclarecida. Vale dizer que medidas de prevenção primária não são consideradas.

\section{O AG no Brasil: necessidades, recursos e perspectivas}

O Sistema Assistencial Público de Saúde no Brasil (SUS) é gigantesco (Tabela 1) e atende entre 60 e $70 \%$ dos 169.590 .693 indivíduos considerados como população residente pelo Censo-2000, o que representa algo em torno de 110 milhões de habitantes. Considera-se que o restante da população tem atendimento de saúde privado. 
Tabela 1

Alguns dados sobre a rede do Sistema Único de Saúde (SUS).

\begin{tabular}{ll}
\hline População residente (censo 2000) & 169.590 .693 \\
Urbana & 137.755 .550
\end{tabular}

Rede ambulatorial (agosto 2001)

Unidades

Complexidade 8 (máxima)

59.057

Produção (procedimentos)

146.855 .217

\begin{tabular}{lrrrrr}
\hline Rede hospitalar & \multicolumn{3}{c}{ Leitos } \\
Região & $\mathrm{N}$ & Universitários & Total & Obstetrícia & Pediatria \\
& 320 & 4 & 25.392 & 5.195 & 5.482 \\
Norte & 1.107 & 41 & 131.728 & 22.724 & 28.955 \\
Nordeste & 473 & 58 & 213.632 & 26.447 & 27.262 \\
Sudeste & 176 & 37 & 76.418 & 10.763 & 13.873 \\
Sul & 244 & 10 & 39.733 & 7.225 & 6.026 \\
Centro-Oeste & 2.320 & 150 & 486 & 72.354 & 81.598 \\
Total & & & & & \\
& & & & 3.144 .547 & \\
\hline Nascidos vivos (1998) & & & & & \\
Em hospital & & & & & \\
De mãe de 35 anos ou + & & & & & \\
\hline
\end{tabular}

Fonte: http.//datasus, gov, br

Sob a égide do conceito "Saúde para todos no ano 2000", a OMS elaborou um documento (OMS-OPAS, 1984) sobre "Prevenção e controle de enfermidades genéticas e defeitos congênitos", o qual considera necessário que os países das Américas iniciem atividades de promoção e proteção da saúde no campo das enfermidades de origem primariamente genética e dos defeitos congênitos que contribuam para a meta fixada.

Se resolvesse implementar essa recomendação, o Ministério da Saúde (MS) teria de pensar que, para atender ao SUS, seria necessário algo em torno de 1.000 médicos especialistas em genética ou treinados para entender as bases genéticas das doenças dos pacientes que atendem (1/100.000 habitantes). A SBGC contabiliza no corrente ano cerca de 200 sócios médicos, dos quais 85 têm título de especialista em genética clínica. Para cada 2 milhões de habitantes seria preciso existir um serviço ou uma rede de serviços que pudesse atender toda a demanda por AG: de anomalias congênitas, passando por doenças metabólicas hereditárias, diagnóstico pré-natal e chegando a testes genéticos moleculares clínicos e préclínicos em doenças degenerativas e câncer. Certamente não há no SUS pelo menos 1 dos 50 serviços (ou rede de) necessários.

Uma situação como esta requer nitidamente uma política de saúde voltada para o problema. O MS tem os instrumentos necessários para implementar tal política e um modelo aparentemente viável é o que está sendo construído a partir da resolução GM/MS 822, de 6 de junho de 2001, que trata do Programa Nacional de Triagem Neonatal (www.saude.gov.br/sas).

Neste modelo os municípios serão responsáveis pela "organização/estruturação/cadastramento ... de pelo menos 1 posto de coleta ... e aos estados compete a organização/estruturação/cadastramento de Serviço(s) de Referência... primeiramente para fenilcetonúria e hipotiroidismo congênito... O acompanhamento dos pacientes diagnosticados com fenilcetonúria ou hipotiroidismo congênito será feito por serviços credenciados que darão assistência integral e simultânea desenvolvida por equipe multiprofissional incluindo orientações clínicas, de risco de recorrência e socioeconômicas". 
Obviamente, o problema é o mesmo de sempre na estrutura do SUS: controle de qualidade. Não há dúvida que aqui também a municipalização é o melhor preventivo contra as habituais mazelas de desvio de função, burocratização ineficiente etc. Campanhas educativas devem ser levadas a efeito no município para envolver a população e orientá-la a vigiar a lisura e qualidade do atendimento.

Uma outra política para incrementar o número de serviços de AG e estimular mais profissionais de saúde a se envolverem com esta prática é a criação de tais serviços na rede assistencial. Como se percebe na tabela 1, a rede hospitalar e ambulatorial do SUS é impressionante: 6.517 hospitais que disponibilizam quase 500.000 leitos e 59.057 unidades de atendimento ambulatorial! Desses hospitais, 150 estão na categoria de hospitais universitários.

A genética médica brasileira poderia responder ao desafio de massificar o atendimento de AG na rede SUS?

Se observarmos os grupos e serviços existentes e a produção científica relacionadas com o AG, a resposta seria "não", por agora; e "sim", a curto e médio prazo.

Na tabela 2 estão escrutinados os 926 trabalhos apresentados nos congressos dos anos de 2000 e 2001 da Sociedade Brasileira de Genética (SBG) e da Sociedade Brasileira de Genética Clínica. Os trabalhos foram organizados por grupos de patologias ou problemas que abordam. Como se percebe, os grupos que os produziram trabalham com agravos genéticos à saúde humana desde os relacionados a problemas reprodutivos, passando pelos perinatais, da infância até a senilidade e chegando ainda à análise de variantes genéticas populacionais potencialmente condicionantes de risco à saúde. Pode-se dizer, portanto, que o universo de problemas genéticos humanos é investigado. Percebe-se que a maior ênfase é dada ao diagnóstico, seja clínico ou com recursos laboratoriais que chegam a testes bioquímicos e moleculares de ponta. A análise dos grupos que produziram esses trabalhos permite também concluir que estão plenamente aptos ao reconhecimento clínico, investigação diagnóstica e condutas pertinentes visando à terapêutica e à prevenção primária, secundária e terciária de pacientes e famílias com as doenças genéticas mais prevalentes no Brasil.

Tabela 2

Trabalhos apresentados (\%) nos congressos anuais da Sociedade Brasileira de Genética (SBG) e na Sociedade Brasileira de Genética Clínica (SBGC).

\begin{tabular}{lcccr}
\hline Grupos de problemas & \multicolumn{2}{c}{ SBG } & \multicolumn{2}{c}{ SBGC } \\
& 2000 & 2001 & 2000 & 2001 \\
\hline 1) AC/Sind/Cli/Epi & 16 & 15 & 54,1 & 53,2 \\
2) AC/Sind/Mol & 27 & 28 & 17,3 & 15,5 \\
3) Sangue-Mol & 10 & 10 & - & 1,5 \\
4) Câncer-Mol & 18 & 19 & 7,1 & 6,0 \\
5) EIM & 1,2 & 1,5 & 14,8 & 16,0 \\
6) DPN & 2 & 1,5 & 2,0 & 2,0 \\
7) AG/Serviços & 0,9 & 1 & 4,8 & 5,1 \\
8) Outros & 25 & 24 & - & - \\
\hline
\end{tabular}

Fontes: anais dos congressos

1) anomalias congênitas e sindromes investigadas pelo método genético-clinico e/ou epidemiológico. inclui citogenética clínica investigada por métodos convencionais; 2) idem por biologia molecular, in clui cito-FISH; 3) doenças do sangue investigadas por métodos moleculares, exclui neoplasias: 4) por cito-FISH e/ou biomol: 5) erros inatos do metabolismo: 6) diagnóstico pré-natal: 7) metodologia no AG, resultados de serviços: 8) principalmente estrutura genética de populaçōes e variantes polimórficas de susceptibilidade a doenças. 
Ao lado desta visão otimista, a constatação é de que os serviços são bons, mas poucos e heterogeneamente distribuídos, refletindo exatamente o problema brasileiro em todas as áreas.

A perspectiva é a de que, com um mínimo de organização e vontade política, é possível em curto espaço de tempo formar, qualificar, treinar e reciclar um número suficiente de profissionais que formarão núcleos em todos os estados e/ou regiões do país a partir dos quais a demanda por AG na rede SUS irá sendo paulatinamente absorvida.

\section{AG no Brasil: proposta de um modelo}

Continuamente vêm sendo apresentadas sugestões e reflexões sobre como a genética médica deveria ser estruturada em países como o Brasil, em especial por meio de publicações no âmbito da Organização Mundial de Saúde/Organização Pan-Americana de Saúde (OMS/OPAS). Um trabalho recente assim (Penchaszadeh et al., 1999) menciona os pontos fundamentais de interesse e oferece o que, a nosso ver, representa o cerne da ação que poderá desencadear um efeito transformador: um Programa de Serviços de Genética organizado e normatizado pelo Ministério da Saúde.

Tais ações poderão ser realizadas no Brasil de maneira relativamente fácil. Temos o Ministério da Saúde, que possui e implementa uma série de programas de complexidade muito maior do que a requerida para os serviços de genética, e existem, distribuídos pelo país, as pessoas e os grupos/serviços que podem ajudar nesta tarefa.

Um grupo técnico do MS ligado à Secretaria de Assistência à Saúde (SAS) pode facilmente levantar a situação do oferecimento de serviços de AG na rede SUS e a maneira de incrementá-lo a curto/médio prazo.

Em linhas gerais, o trabalho seria o de localizar as Unidades de Atendimento ideais em cada região/estado. Esses locais seriam aqueles nos quais alguma ação de AG é desenvolvida, como hospitais universitários ou unidades de atendimento com a abrangência populacional adequada. Nesses locais devem ser identificados os profissionais já envolvidos com o AG; caso não existam, é recomendável que se estimulem os interessados. Outra medida é pensar numa equipe básica composta por médico, psicólogo, laboratoristas para a área de citogenética e bioquímica de erros inatos do metabolismo. Paralelamente, seriam localizados grupos, centros e serviços de AG que tenham infra-estrutura para formação em genética médica básica, treinamento clínico, procedimentos laboratoriais em citogenética convencional, triagem e cromatografias para erros inatos do metabolismo e eletroforese de hemoglobinas. As especificidades e necessidades de cada região seriam consideradas. Encontrando quem queira aprender e quem queira ensinar, este programa do MS deve prover os meios ou procurar viabilizá-los para que esses agentes se encontrem e o treinamento ocorra. É crucial que o SUS monte um sistema capacitado para absorver essa mão de obra. Ou seja, esse pessoal tem que ser contratado pelo SUS ou remunerado por ele. Serviços e procedimentos remunerados significam serviços oferecidos: dentro da complexidade de hospitais universitários é muito fácil criar unidades que comportem esses profissionais. Equipar laboratórios de citogenética ou de bioquímica para erros inatos do metabolismo dentro de serviços de patologia clínica, facílimo e barato.

O panorama da assistência em genética médica no Brasil começará certamente a mudar se tal modelo permitir a criação de pelo menos uma unidade de AG em cada um dos 150 hospitais universitários, com uma equipe minimamente constituída pelos seguintes profissionais: geneticista-clínico, citogeneticista, bioquímico treinado em exames básicos para erros inatos do metabolismo e psicólogo e/ou assistente social. Neste momento, parece precipitado pensar no $A G$ em equipes de atenção primária à saúde, mesmo em países desenvolvidos (Greendale \& Pyeritz, 2001). Portanto, parece ser mais adequado organizar serviços em unidades secundárias ou terciárias com pessoal especializado. Em etapas subseqüentes, esta prática irá sendo transferida para a atenção primária, o que possibilitará atingir o universo do atendimento (Guttmacher et al., 2001). 
É claro que enquanto isso está sendo feito, outras ações devem ser realizadas para permitir a continuidade e aprimoramento na oferta de serviços de AG.

Um deles diz respeito ao ensino da genética médica, seja nos cursos de graduação em biomedicina, psicologia etc. ou na formação de profissionais para atuar no AG.

Do ponto de vista da formação de médicos, não há dúvida que os Programas de Residência Médica para graduados e de Cursos de Especialização para egressos de programas de Residência Médica são o melhor método. Esses programas existem e funcionam regularmente, sendo apenas necessário que mais programas se estruturem e passem a oferecer este treinamento.

Profissionais não-médicos, principalmente biólogos e biomédicos, dedicam-se ao AG em serviços universitários brasileiros ou institutos de pesquisas. Esses profissionais são quase sempre pós-graduados (PG) em programas de genética humana/genética médica, com interesse primordial em certas doenças ou grupos de doenças genéticas. Nos Estados Unidos e no Canadá, instituiu-se a carreira de "aconselhador genético", profissional que tem trabalhado dentro de equipes de AG. Programas de PG têm a finalidade de formar pesquisadores, portanto seria conveniente oferecer cursos de especialização para graduados em cursos pertencentes à área de saúde que desejem envolver-se com a assistência em AG. Recentemente, o sistema de PG brasileiro, via CAPES, tem estimulado a abertura de Mestrados Profissionalizantes, cuja aceitação tem gerado polêmica. Na área do AG, talvez fossem uma maneira de atender ao que parece uma crescente demanda de profissionais já atuantes em suas áreas e que se sentem atraídos por esta atividade: dentistas, psicólogos, enfermeiros, assistentes-sociais, biólogos, biomédicos etc.

Outro ponto de máxima importância diz respeito ao envolvimento da sociedade com os problemas de saúde geneticamente determinados. Possivelmente é a ação mais eficiente para fazer com que mais pessoas tenham essa "educação para a saúde genética", incentivando, apoiando e ajudando a criar associações e grupos de pacientes, famílias e interessados em certa doença ou grupo de doenças genéticas. Não há dúvida que quanto maior for o envolvimento da comunidade, melhores e mais eficazes serão as ações de AG (Ramalho et al., 1999). Experiências desse tipo existem no Brasil, tanto na forma de associações como em trabalhos comunitários. Essas ações, que vêm se multiplicando em todo o mundo, foram mais recentemente englobadas no que tem sido chamado de "genética comunitária" (Modell \& Kuliev, 1998; Ramalho \& Silva, 2000). Cada serviço de AG poderia criar sua genética comunitária, elegendo os problemas mais prevalentes e criando grupos de usuários para discuti-los em conjunto.

\section{Conclusões}

Neste trabalho procuramos dar uma visão bastante prática de como estão os Serviços de Aconselhamento Genético no Brasil no que diz respeito ao atendimento da população. O que temos é de boa qualidade, mas insuficiente. O número desses serviços pode ser aumentado mediante a adoção de algumas medidas facilmente implementadas:

1) inserir o AG na rede do Sistema Único de Saúde do Brasil, criando pelo menos 150 serviços, um em cada hospital universitário da rede;

2) estimular e oferecer condições para que médicos e outros profissionais da saúde lotados nesses hospitais ou por eles contratados façam uma formação em genética médica/genética clínica e em métodos diagnósticos complementares;

3) identificar grupos e serviços que possam formar, treinar e reciclar esses profissionais em programas de aperfeiçoamento/especialização;

4) criar um Grupo Técnico no Ministério da Saúde para organizar ações visando atingir os objetivos acima; 
5) estimular os serviços de AG existentes para apoiar e tomar iniciativas visando a ações de genética comunitária;

6) estimular as sociedades científicas a organizar grupos visando oferecer sugestões para incrementar o ensino da genética médica em cursos de graduação da área da saúde.

\section{Referências bibliográficas}

Biesecker BB \& Peters KF 2001. Process studies in genetic counseling: peering into the black box. American J ournal of Medical Genetics 106: 191-198.

Brunoni D 1997. Estado atual do desenvolvimento dos serviços de genética médica no Brasil. Revista Brasileira de Genética 20 (1, supl.): 11-23.

Brunoni D 1999. O médico especialista em genética. Médicos II (6): 49-53.

Costa T, Scriver CR \& Childs B 1985. The effect of Mendelian disease on human health: A measurement. American J ournal of Human Genetics 21:231-236.

Epstein CJ 1975. Genetic couseling: statement of the American Societay f Human Genetics ad hoc Comittee on Genetic Counseling. American Journal of Human Genetics 27(2): 241242.

Gjorup T, Hendriksen C, Bugge TM \& Jensen AM 1989. A bedside study I: the influence of physical findings on the global assessment. Journal of Internal Medicine 226: 123-126.

Greendale K \& Pyeritz RE 2001. Empowering primary care health professionals in medical genetics: how soon? How fast? How far? American Journal of Medical Genetics 106: 223232.

Grupo de Trabalho 1997. Conclusiones y recomendaciones de la reunión de expertos en servicios de genética médica en América Latina. Brazilian Journal of Genetics 20 ( 1 suppl): 171-174.

Guttmacher AE, Jenkins J \& UhImann W 2001. Genomic medicine: who will practice it? A call to open arms. American J ournal of Medical Genetics 106: 216-222.

Hampton J R, Harrison MJ, Mitchell JR, Pritchard JR \& Seymour C 1975. Relative contribution of history-taking, physical examination and laboratory investigation to diagnosis and management of medical outpatients. British Medical J ournal 2:486-489.

Marques-de-Faria AP, Brunoni D \& Ferraz VEF 2000. Aconselhamento genético. Documento encaminhado ao Conselho Federal de Medicina.

Marques-de-Faria AP, Ferraz VEF, Acosta AX \& Brunoni D 2001. Clinical genetics in the developing countries: the Brazilian situation. Genetics in Medicine (a ser submetido).

Ministério da Saúde. Indicadores de Saúde, 2001. http://datasus.gov.br

Ministério da Saúde 2000. http://saude.gov.br/sas

Modell B \& Kuliev A 1998. The history of community genetics: The contribution of the haemoglobin disorders. Community Genetics 1:3-11.

OMS-OPAS. Publicação Científica, n.460, 1984. 
Penchaszadeh VB, Christianson AL, Giugliani R, Boulyjenkov V \& Katz M 1999. Services for the prevention and management of genetic disorders and birth defects in developing countries. Community Genetics 2: 196-201.

Pergament E 1997. A clinical geneticist perspective of the patient-physician relationship, $p$. 92-107. In Rothstein MA (ed.). Genetic secrets: protecting privacy and confidentiality in the genetic era. Yale University Press, New Haven e Londres.

Ramalho AS, de Paiva e Silva RB, Teixeira RC \& Compri MB 1999. Hemoglobin screening: response of a Brazilian community to optional programs. Cadernos de Saúde Pública 15(3): 591-595.

Ramalho AS \& de Paiva e Silva RB 2000. Community genetics: a new discipline and its application in Brazil. Cadernos de Saúde Pública 16(1): 1. 